\title{
Desconocimiento sobre la Magnitud del Aborto en Colombia
}

\section{Evidencia de seis Encuestas Realizadas entre 1965 y 1980}

\author{
Dr. Alberto Rizo G., MD. D., M.S.P. \\ Dra. Elena Prada Salas, Soc. M.S.C. \\ XIV Congreso Colombiano de Obstetricia y Ginecología \\ Cartagena - 1981
}

El aborto puede ser estudiado de varias maneras: mediante el análisis de las estadísticas vitales, en particular en aquellos países en donde la práctica del aborto es legal; mediante el estudio de las estadísticas de mortalidad materna por aborto, de la mortalidad fetal etc. Igualmente se estudia el aborto al analizar las estadísticas hospitalarias (egresos por aborto) y las estadísticas sobre juicios a personas a quienes se las ha acusado de haberse provocado un aborto o permitido que otro se lo practique.

Además de los estudios de las estadísticas anteriormente mencionadas, también las encuestas permiten conocer el número y características de los abortos que se producen en un lugar. Al estudiar las estadísticas y las encuestas como las kap, la información se obtiene sobre hechos sucedidos, es decir retrospectivamente. Pero no solo en forma retrospectiva las encuestas proporcionan in-

Cuadro No. 1

\section{ABORTO}

METODOS PARA ESTUDIAR SU INCIDENCIA

\section{RETROSPECTIVOS}

1. Estadísticas vitales (Registro del aborto en países que han legalizado su práctica - Es tadísticas de Mortalidad por aborto).

2. Otras estadísticas (Estadísticas hospitalarias y Instituciones de Salud - Juicios legales).

3. Estudios especiales (Encuestas tipo KAP Encuestas que utilizan técnicas como la RRT)

\section{PROSPECTIVOS}

1. Entrevistas mediante encuestas repetidas a una misma comunidad por tiempo determinado.

2. Detección de embarazos/abortos mediante determinación de HCG (hormona gonadotrofina coriónica).

formación sobre aborto. También prospectivamente puede obtenerse dicha información. 
Una muestra de mujeres en edad fértil seguidas durante un tiempo determinado y encuestadas periódicamente (1-3 meses) en relación a embarazos/ abortos, da igualmente información que muchos consideran menos afectada de subregistro que la información obtenida en forma retrospectiva. A fin de mejorar aun más el registro, se ha propuesto seguir en forma prospectiva los embarazos que se producen en una muestra de mujeres en edad fértil, a las cuales se practican periódicamente análisis para detectar, por técnicas de laboratorio muy sensibles y altamente específicas, la hormona gonadotrofina coriónica, la cual siempre está presente cuando existe un embarazo. Desafortunadamente el elevado costo de tales determinaciones hormonales hace difícil de realizar en nuestro medio este estudio.

Con ocasión del Congreso Colombiano de Obstetricia y Ginecología celebrado hace dos años en la ciudad de Bogotá, uno de los autores del presente estudio realizó un análisis de 78 publicaciones sobre aborto aparecidas en Colombia entre 1960 v 1980. La mayoría de tales publicaciones $(36 \%)$ reportaban información sobre el aborto tal como se presenta en el hospital. La mayor parte de los estudios publicados en 20 años eran de tipo transversal, descriptivos y utilizaron información obtenida retrospectivamente. Se señalaron las limitaciones de tales estudios y se propuso revisar otras metodologías para mejorar la calidad de la información obtenida.

Los estudios hospitalarios, es bien conocido, solo proporcionan información sobre una parte de los abortos que se suceden en determinado lugar. Cuando el aborto cursa sin complicaciones, no se hospitaliza y muchos abortos aún no requieren atención médica.

Aun cuando en Colombia no se han realizado encuestas específicas sobre el aborto, si han sido efectuados varios estudios que han incluido preguntas que permiten conocer, en forma retrospectiva, algunos aspectos del aborto.

Contrario a lo que uno pudiera creer, poca ha sido la contribución al conocimiento sobre el aborto en Colombia que estas encuestas han aportado. Sin conocer aún los resultados de la última encuesta nacional de prevalencia de uso de anticonceptivos realizadas en 1980 y los resultados de la encuesta nacional de salud del $M$. del S.P. realizada entre 1977-1980, solo dos de las 7 encuestas publicaron resultados que permiten tener una perspectiva del aborto diferente a la que dan los estudios realizados en el hospital.

Se observan los resultados sobre aborto que dieron la investigación nacional de morbilidad realizada en 1965 por el Ministerio de Salud y la Asociación Colombiana de Facultades de Medicina: 136.1 abortos y 165 muertes fetales por c/1000 embarazos. La primera encuesta nacional de fecundidad, realizada 4 años más tarde por Ascofame y Celade, muestra 126 muertes fetales por cada 1000 embarazos en mujeres entre 15 y 19 años, calculadas para el período 19641968.

Se incluye el comentario que hacen los autores de la publicación "hechos demográficos" del estudio de recursos humanos para la salud y educación médica en Colombia, Drs. Carlos Agualimpia y Aurelio Pabón en relación a los resultados obtenidos:

“El riesgo de aborto podría estar sub-estimado si se considera que una fracción de los abortos ocurridos realmente fueron ocultados al entrevistador y que esa omisión ha sido proporcionalmente mejor que la de embarazos".

En Nov/73, la Socióloga Elena Prada, Co-autora de este trabajo, afirmaba sobre 
Cuadro No. 2

GRANDES ENCUESTAS* EN LAS AREAS DE SALUD/POBLACION QUE INCLUYERON PREGUNTAS SOBRE EL ABORTO (1965-1980)

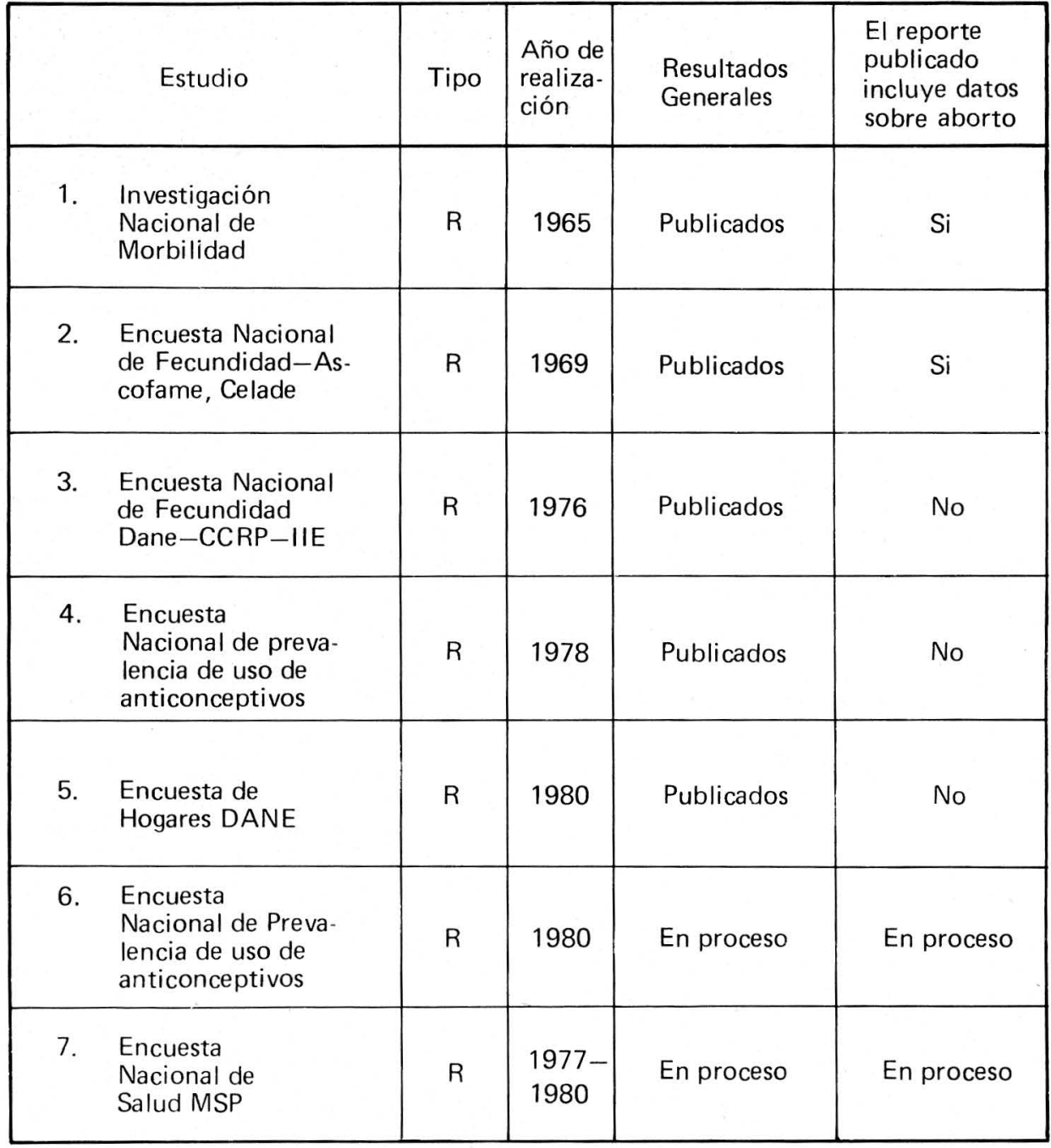

R: Retrospectivo

No se incluye:

- Estudio de Mortalidad urbana (Puffer R - Griffith G.W. OPS/67) 


\section{INCIDENCIA DEL ABORTO SEGUN LOS RESULTADOS PUBLICADOS \\ POR DOS ENCUESTAS EN COLOMBIA (1965-1969)}

\begin{tabular}{|l|c|c|c|}
\hline \multicolumn{1}{|c|}{ Encuesta } & Tipo & Año & \multicolumn{1}{|c|}{ Resultados } \\
\hline $\begin{array}{l}\text { Investigación Nacional } \\
\text { de Morbilidad } \\
\text { (MSP - Ascofame) }\end{array}$ & $\mathrm{R}$ & 1965 & $\begin{array}{l}\text { Se encontraron 136.1 abortos } \\
\text { por cada 1000 embarazos y } \\
165 \text { defunciones fetales } \times 1000 \\
\text { embarazos }\end{array}$ \\
\hline $\begin{array}{l}\text { Encuesta Nacional* } \\
\text { de fecundidad } \\
\text { (Ascofame-Celade) }\end{array}$ & $\mathrm{R}$ & 1969 & $\begin{array}{l}\text { Para el período 1964-1968 } \\
\text { las tasas de pérdida* oscilaron } \\
\text { alrededor de 126 x cada 1000 } \\
\text { embarazos en grupos de 15 }- \\
\text { 49 años. }\end{array}$ \\
\hline
\end{tabular}

* Incluye nacidos, muertos, abortos espontáneos y abortos provocados en este cálculo.

los resultados acerca del aborto que dió la primera encuesta de fecundidad: "las tasas de pérdida no ofrecen mayor confiabilidad, puesto que en su cálculo está incidiendo el factor olvido y su magnitud es difícil de calcular".

Los autores del presente estudio consideramos que, de conocerse los resultados de las encuestas restantes mencionadas al comienzo, estarían acompañados de comentarios parecidos a los anteriormente citados.

Esta es una muestra del formulario de entrevistas domiciliarias que utilizó la encuesta nacional de morbilidad en 1965.

Las preguntas 14 a y 14 b daban al entrevistador la posibilidad de indagar acerca del número de hijos vivos de toda mujer entrevistada entre $15-54$ años y respecto de los embarazos y abortos de las mujeres referidas al período enero/ 1965 y el momento en el cual la mujer fue entrevistada. Obsérvese en la Tabla la 2a y la última columna correspondiente a embarazos.

La historia de nacimientos permite a los investigadores determinar con alguna precisión la ocurrencia de estos eventos en la vida de una mujer. Es utilizada con bastante frecuencia en investigaciones de tipo demográfico en todo el mundo.

Bajo el encabezamiento "otros emba-

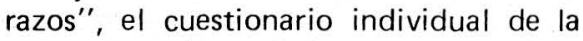
encuesta nacional de fecundidad permite al encuestador obtener información sobre aquellos embarazos que terminaron 


\section{ESTUDIO DE RECURSOS HUMANOS PARA LA SALUD Y EDUCACION MEDICA EN COLOMBIA FORMULARIO DE ENTREVISTAS DOMICILIARIAS HECHOS DEMOGRAFICOS INVESTIGADOS*}

\begin{tabular}{|c|c|c|c|c|}
\hline & $\begin{array}{l}\text { Pregurtu para TODA MUJER entre } 15 \text { y } 54 \\
\text { años de edad. }\end{array}$ & $14 a-$ Cuäntos hijos vivos he tenido usted? & $\begin{array}{l}\text { Número de hijos } \\
\text { No aplicable } \square\end{array}$ & Nínguno $\square$ \\
\hline 14b. & $\begin{array}{l}\text { Pregunte para TODA MUJER entre } 15 \text { y } 54 \\
\text { años de edad. } \\
\text { Si lo respuesta es SI. proceda a llenar la } \\
\text { TABLA I, en la ültima página. }\end{array}$ & $\begin{array}{l}\text { 14b - Durante el período comprendido entre el } 10 . \\
\text { de enero de } 1965 \mathrm{v} \text { el momento presente, ha } \\
\text { tenido usted algún hijo vivo, una pérdida o } \\
\text { aborto, o un nacido muerto? }\end{array}$ & Si $\square$ i & No. $\square 2$ \\
\hline $14 a$. & $\begin{array}{l}\text { Pregunte para TODA MUJER entre } 15 \text { y } 54 \\
\text { años de edad. }\end{array}$ & 14a. Cuántos hijos vivos ha tenido usted? & $\begin{array}{l}\text { Número de hijos } \\
\text { No aplicable }\end{array}$ & Ninguno $\square$ \\
\hline $14 \mathrm{~b}$ & $\begin{array}{l}\text { Pregunte para TODA MUJER entre } 15 \text { y } 54 \\
\text { años de edad. } \\
\text { Si la respuesta es SI, proceda a llenar la } \\
\text { TABLA I, en la útína página. }\end{array}$ & $\begin{array}{l}\text { 14b. Durante el periodo comprendido entre la Se. } \\
\text { mana Santa del año pasado y el momento } \\
\text { presente, ha tenido usted aigún hijo vivo, una } \\
\text { pérdida o aborto, o un nacido muerto? }\end{array}$ & si $\square$ ' & No $\square 2$ \\
\hline
\end{tabular}

\begin{tabular}{|c|c|c|c|c|c|c|c|c|c|c|c|c|c|c|}
\hline \multicolumn{15}{|c|}{ TABLA No. 1 - EMBARAZOS TERMINADOS } \\
\hline \multirow{3}{*}{ 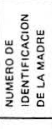 } & \multicolumn{6}{|c|}{ EMEARAZOS } & \multicolumn{4}{|c|}{ paAtos } & \multicolumn{4}{|c|}{ PARA NACIDOS VIVOS } \\
\hline & \multirow{2}{*}{$\begin{array}{l}\text { CUAL FUE LA } \\
\text { DURACION EN } \\
\text { MESES ? }\end{array}$} & \multicolumn{2}{|c|}{$\begin{array}{l}\text { TUVO ATENCION } \\
\text { MEDICA? }\end{array}$} & \multicolumn{3}{|c|}{$\begin{array}{c}\text { COMO TERMINO EL } \\
\text { EMBARAZO? }\end{array}$} & \multirow{2}{*}{\multicolumn{3}{|c|}{$\begin{array}{l}\text { OUIEN ATENOOO EL PARTO } \\
\text { (OABORTO)? }\end{array}$}} & \multirow{2}{*}{ DONDE OCURAIO ? } & \multicolumn{2}{|c|}{$\begin{array}{l}\text { FUE } \\
\text { REGISTRADO }\end{array}$} & \multicolumn{2}{|c|}{$\begin{array}{l}\text { FUE } \\
\text { BAUTTZZADO }\end{array}$} \\
\hline & & st & No & Nacido & Nocido & АBORTO & & & & & si & No & si & No \\
\hline & & & & & & & $\begin{array}{l}\text { Mosico } \\
\text { Entermere } \\
\text { Otros }\end{array}$ & $\begin{array}{l}\square \\
\square s \\
\square s\end{array}$ & Comedrons $\square^{2}$ & 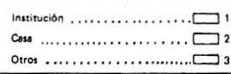 & & & & \\
\hline & & & & & & & $\begin{array}{l}\text { Medico } \\
\text { Entermera } \\
\text { Oros }\end{array}$ & $\begin{array}{l}\square: \\
\square:\end{array}$ & $\begin{array}{l}\text { Comodrons } \\
\text { Tegus }\end{array}$ &  & & & & \\
\hline & & & & & & & $\begin{array}{l}\text { Medico } \\
\text { Entiemers } \\
\text { Otror }\end{array}$ & $\begin{array}{l}\square \\
\square\end{array}$ & comadrons $\square_{\text {Tegus }}^{2}$ & 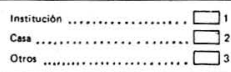 & & & & \\
\hline & & & & & & & & & 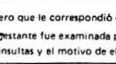 & 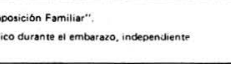 & & & & \\
\hline
\end{tabular}

en abortos, muertes fetales tardías y otros nacimientos no referidos en la hoja anterior. Igualmente se indaga sobre maniobras para interrumpir el embarazo.

Podrán darse cuenta con esta parte del cuestionario, acerca de las dificultades que se le presentan al investigador cuan. do se indaga sobre embarazos que han terminado en aborto, su duración, la presencia o ausencia de maniobras etc.

Podría pensarse que los resultados sobre abortos obtenidos en cada una de las 2 encuestas a que nos hemos referido están sub-estimados por cuanto tales encuestas no tenían el estudio del aborto como objetivo específico de la encuesta: la una era general sobre salud y la otra encuesta era de tipo demográfico en general.

Se incluyen los resultados de encuestas específicas sobre aborto realizados a mediados de la década de los años 60 en algunas ciudades colombianas. La proporción abortos/embarazos por 100 es bastante parecida a la proporción encontrada en el estudio nacional de morbilidad y en la 7a. encuesta de fecundidad.

Se mencionan algunos de los factores por los cuales se presenta subregistro 
Cuadro No. 4

\section{ENCUESTAS ESPECIFICAS \\ DATOS SOBRE PREVALENCIA \\ DEL ABORTO EN ALGUNAS CIUDADES COLOMBIANAS}

(1964)

\begin{tabular}{|l|c|c|c|}
\hline Ciudad & Embarazos & Abortos & $\begin{array}{l}\text { Abortos/ } \\
\text { Embarazos \% }\end{array}$ \\
\hline Cali & 8981 & 1303 & 14.5 \\
\hline Candelaria & 776 & 109 & 14.0 \\
\hline Manizales & 8487 & 1351 & 15.9 \\
\hline Popayán & 4911 & 571 & 11.6 \\
\hline
\end{tabular}

Ref: Estudios sobre aborto en Cali - Candelaria, Manizales y Popayán - Planificación Familiar Motivación Comunicación - Valoración Ascofame - Bogotá.

del aborto en las encuestas que obtienen información en forma retrospectiva: naturaleza ilegal del aborto; temor de la entrevistada a ser censurada por el entrevistador; por fallas en la memoria especialmente en mujeres viejas; por dificultades en la comprensión de la entrevistada del término aborto, por una inadecuada definición del mismo, por la tendencia del ser humano a olvidar los hechos leves y recordar solo los complicados $y$ en fin porque la entrevistada simplemente no desea revelar a un entrevistador hechos que tocan muy de cerca su vida intima.

Quizás en América Latina sea Chile el país en donde más se ha estudiado el aborto. En estudios realizados la proporción de abortos/embarazos por 100 fue más alta cuando se obtuvo en forma prospectiva. En cambio la tasa de abortos por $100 \mathrm{MEF}$ no fue tan elevada como podría esperarse.

Esto nos demuestra que aun cuando la información sobre aborto que se obtiene prospectivamente puede mejorar en calidad respecto a la que se obtiene en forma retrospectiva tampoco es la ideal según la opinión de quienes mejor conocen el estudio del aborto.

Precisamente el dr. Christopher Tietze de Population Council en New York es una de esas autoridades. Un modelo matemático ideado por el Dr. Tietze nos muestra que podría esperarse en relación con la proporción de abortos/100 partos en comunidades que utilizan en forma variable la anticoncepción o no la utilizan.

\section{Gráfica No. 1}

\section{PROPORCION \\ DE ABORTOS/PARTOS}

SEGUN MODELO TEORICO DE TIETZE

1

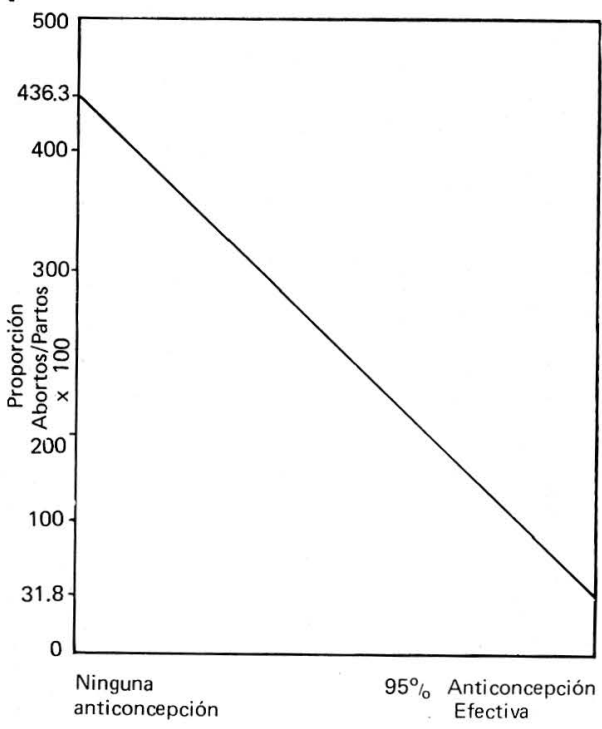

Es posible que la línea recta del diagrama no corresponda a lo real y que antes que una recta, la línea sea curva y presente no una sino varias sinuosidades. En alguno de los puntos debe encontrarse nuestro país. Desafortuna- 


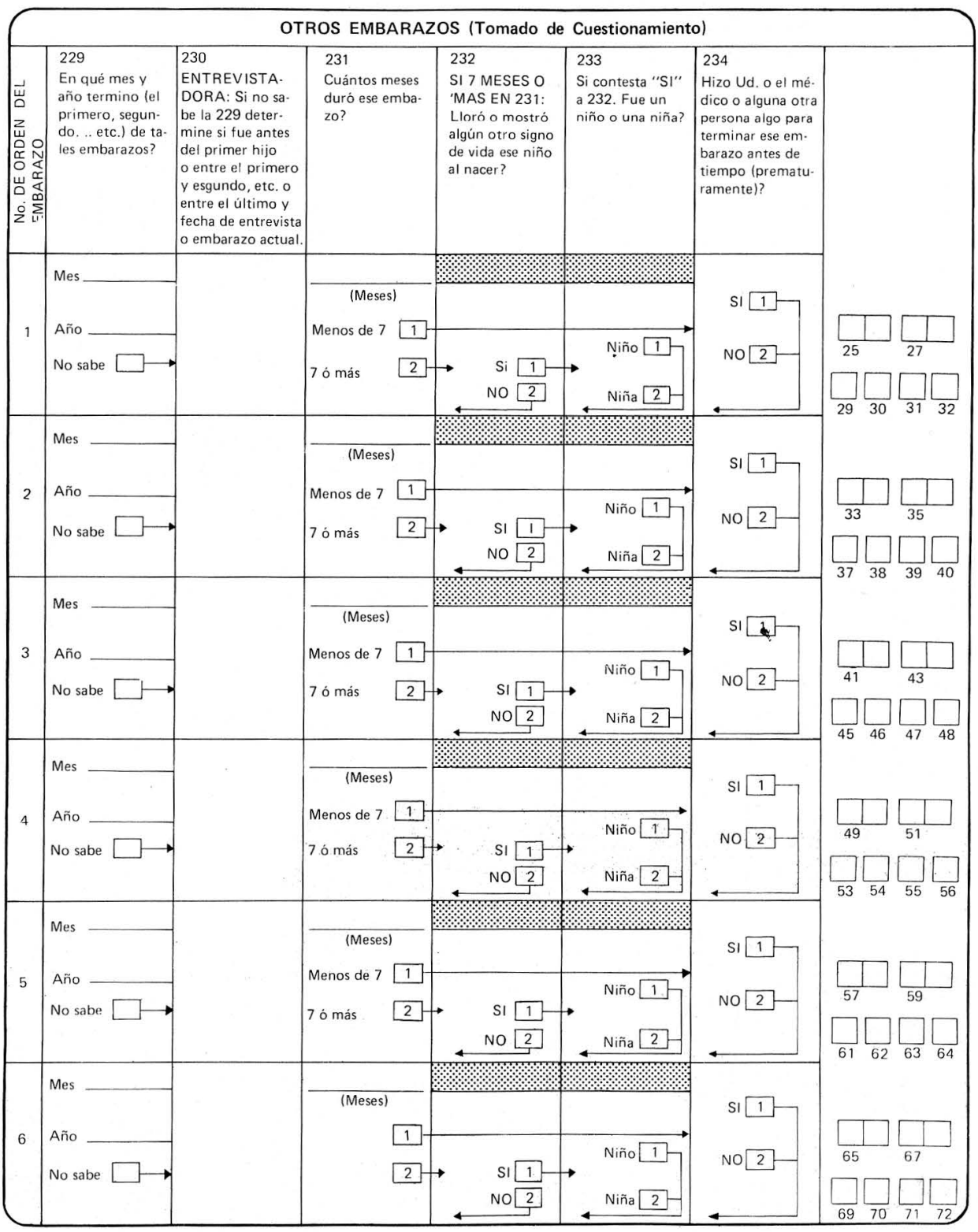


damente no lo conocemos aún $y$ es seguro que no lo vamos a conocer muy pronto. No conocemos de estudios ni en ejecución ni en proyecto que tengan en mente despejar la incógnita existente: desconocemos aún la verdadera magnitud del aborto en nuestro medio y no se hacen esfuerzos para establecer su número y caracter isticas.

El presente trabajo ha tenido como objetivos:

1. Contribuir al conocimiento sobre las ventajas y limitaciones que ofrecen las encuestas retrospectivas para estudiar el aborto en nuestro medio.

2. Señalar la importancia que tiene el conocer la información que existe sobre el aborto inducido.

3. Promover el interés de estudiantes residentes, profesores y demás profesionales de la salud por el estudio del aborto inducido en Colombia.

Muchos de los asistentes van posiblemente a creer que si las encuestas presentan limitaciones lo mismo que los estudios hospitalarios del aborto, pocas son entonces las opciones que se ofrecen al investigador que se interesa por estudiar al aborto. La combinación de una encuesta y un estudio hospitalario hechos con idéntico período de referencia, la una retrospectivamente $y$ el otro en forma prospectiva, parecen teritr un futuro promisorio.

El grupo para el estudio del aborto inducido de la OMS en Ginebra ha preparado recientemente un documento describiendo la metodología.

\section{Conclusiones y recomendaciones}

Ante las limitaciones de los estudios hospitalarios solos y de las encuestas retrospectivas aisladas para conocer la magnitud y características del aborto inducido se recomienda:

1. Estimular el interés de las autoridades de Salud en Colombia para que se aporten los recursos económicos necesarios a fin de continuar el estudio del aborto inducido en el pa ís.

2. Promover la realización de investigaciones que combinen en forma paralela el estudio del aborto en el hospital (prospectivo) con encuesta retrospectiva en una muestra aleatoria de la misma comunidad segun lo recomienda la Organización Mundial de la Salud.

\section{IGNORANCE OF THE MAGNITUDE OF ABORTION IN COLOMBIA: EVIDENCE OF SIX USRVEYS CONDUCTED BETWEEN 1965-1980}

The main objectives of this article are:

1. To contribute to a better understanding of the advantages and disadvantages of retrospective surveys to study the abortion problem in Colombia.
2. To point out the importance of obtaining all existing information on induced abortion.

3. To promote the interest of students, residents, faculty and other health professionals in the study of induced abortion in Colombia. 
Taking into consideration the limitations of hospital studies per se, and of isolated retrospective surveys in the research of amplitude and characteristics of induced abortion, we recommend:

1. To stimulate the interest of health officials of Colombia, in order to allocate the necessary funding to pursue research on induced abortion in this country.

2. To promote the kind of methodology that combines analysis of abortion in hospitals (prospective), and random retrospective surveys of the same community, as recommended by the World Health Organization. 